\title{
Analytical energy loss distribution for accurate high resolution depth profiling using medium energy ion scattering
}

\author{
R. P. Pezzi, ${ }^{1,2, a)}$ C. Krug $^{2}$ P. L. Grande, ${ }^{2}$ E. B. O. da Rosa, ${ }^{2}$ G. Schiwietz, ${ }^{3}$ and \\ I. J. R. Baumvol ${ }^{2,4}$ \\ ${ }^{1}$ IBM Research Division, T. J. Watson Research Center, P.O. Box 218, Yorktown Heights, New York 10598, \\ USA \\ ${ }^{2}$ Instituto de Física, UFRGS, Av. Bento Gonçalves, 9500, 91509-900 Porto Alegre, Rio Grande do Sul, \\ Brazil \\ ${ }^{3}$ Hahn-Meitner-Institut, Abteilung SF4, Glienicker Strasse 100, 14109 Berlin, Germany \\ ${ }^{4}$ Centro de Ciências Exatas e Tecnologia, UCS, Av. Francisco G. Vargas, 1130, 95070-560 Caxias do Sul, \\ Rio Grande do Sul, Brazil
}

(Received 4 February 2008; accepted 10 April 2008; published online 25 April 2008)

\begin{abstract}
An analytical approach to ion energy loss distributions capable of simplifying medium energy ion scattering (MEIS) spectral analysis is presented. This analytical approach preserves the accuracy of recent numerical models that evaluate energy loss effects overlooked by standard calculations based on the Gaussian approximation. Results are compared to first principle calculations and experimental MEIS spectra from 0.2- to 1.5-nm-thick $\mathrm{HfO}_{2}$ films on $\mathrm{Si}$, supporting the application of this analytical model for proton scattering in the kinetic energy range from 100 to $200 \mathrm{keV}$.

(C) 2008 American Institute of Physics. [DOI: 10.1063/1.2918443]
\end{abstract}

The depth distribution of chemical elements near the surface of solids is of major relevance in many aspects of science and technology. In principle, it can be quantitatively determined on an absolute scale (i.e., without reference to standards) by using ion scattering. Subnanometric depth resolution can be achieved in near-surface regions through the combined use of high resolution energy analyzers ${ }^{1}$ and incident ions with a kinetic energy corresponding to the maximum stopping power of the sample. ${ }^{2}$ This setup corresponds to the technique of medium energy ion scattering (MEIS), ${ }^{3}$ which became a natural solution for material characterization in current microelectronics research. ${ }^{4}$

Quantitative interpretation of MEIS spectra demands an accurate description of ion energy loss distributions as a function of depth of the backscattering event in the sample. $^{5-11}$ Owing to its simplicity and to its analytical expression, Gaussian ion energy loss distribution functions are used aiming at the high depth resolution that can, in principle, be provided by MEIS. ${ }^{12-17}$ The use of Gaussian distributions is supported by the central limit theorem, according to which the energy loss is normally distributed if the number of energy loss events (atomic collisions) is large. ${ }^{11,18}$ This condition, however, is not satisfied in the characterization of near-surface, nanoscale structures, where only a small number of energy loss events comes into play. ${ }^{4,19}$ A more accurate, stochastic approach to ion energy loss distributions was recently demonstrated ${ }^{5}$ to be necessary in this case, which takes into account also electronic excitations of the target atom as obtained from ab initio calculations. However, the computational budget required for these calculations prevents their widespread application to data analysis.

In this letter, we propose a semiempirical, analytical solution of the Bothe-Landau equation ${ }^{11}$ that can conveniently replace both the Gaussian approximation and the more complete numerical approach of Ref. 5 in data analysis software. The validity of the present analytical energy loss distribution

${ }^{a)}$ Electronic mail: rppezzi@us.ibm.com. model is verified by comparison with full $a b$ initio simulations $^{6}$ and experimental MEIS spectra determined from $\mathrm{HfO}_{2}$ films on $\mathrm{Si}$ in the thickness range from 0.2 to $1.5 \mathrm{~nm}$.

It can be shown that for ions interacting with amorphous or polycrystalline targets, the energy loss law is governed by Poisson statistics. ${ }^{9,10}$ The distribution of energy loss $\Delta E$ as a function of trajectory length $x$ in the target can then be written as

$$
F(\Delta E, x)=\sum_{n=0}^{\infty} P_{n}(x) f^{* n}(\Delta E),
$$

where $P_{n}(x)=\left[(m x)^{n} / n !\right] e^{-m x}$ are Poisson coefficients regarding $n$ cumulative collisions at an average of $m$ collisions per unit trajectory length, $f^{* n}(\Delta E)$ represents $n$ self-convolutions of the normalized probability of energy loss $f(\Delta E)$ $=\sigma(\Delta E) / \int \sigma(\eta) d \eta$, and $\sigma(\Delta E)$ being the cross section of energy loss $\Delta E$ in a single atomic collision. Details can be found in Refs. 5, 9, and 10 .

The normalized probability of energy loss (or its selfconvolutions) cannot be experimentally determined, justifying the use of $a b$ initio calculations as in the coupled channels approach. The method consists of solving the timedependent Schrödinger equation by using an expansion of the electronic wave function in terms of Hartree-Fock-Slater atomic orbitals for each initial state and target element. ${ }^{6}$ It yields the absolute probability of single electronic excitations due to the time-dependent potential caused by the incident projectile as a function of impact parameter. The energy loss cross section $\sigma_{i}(\Delta E)$ is then determined by averaging over all possible impact parameters for each target element $i$. The stopping power $d E / d x$ and energy straggling constant $d W^{2} / d x$ in convenient units are the first and second moments of $\sigma_{i}(\Delta E)$ multiplied by the number of target atoms per unit of volume, respectively; Bragg's law is assumed to hold for compound targets. In the case of present interest, namely, $100 \mathrm{keV} \mathrm{H}^{+}$ions traversing a $\mathrm{HfO}_{2}$ matrix with density of 


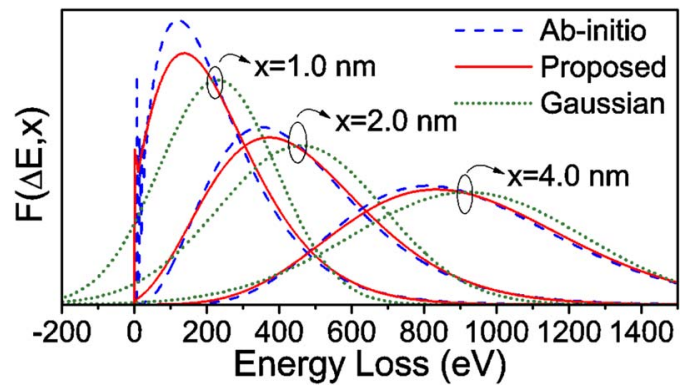

FIG. 1. (Color online) Energy loss distributions for $100 \mathrm{keV} \mathrm{H}^{+}$ions crossing 1.0, 2.0, and $4.0 \mathrm{~nm}$ of $\mathrm{HfO}_{2}$ as determined by ab initio (dashed lines), Eq. (3) (solid lines), and the standard Gaussian approximation (dotted lines).

$9.5 \mathrm{~g} \mathrm{~cm}^{-3}$, we determined $d E / d x=230 \mathrm{eV} / \mathrm{nm}$ and $d W^{2} / d x=2.61 \times 10^{4} \mathrm{eV}^{2} / \mathrm{nm}$. Although these figures agree to within $20 \%$ with those from more accurate estimates such as SRIM (Ref. 20) and Chu, ${ }^{21}$ they were adopted for consistency among the calculations presented below.

The dashed lines in Fig. 1 depict the energy loss distribution according to ab initio calculations for $100 \mathrm{keV} \mathrm{H}^{+}$ ions after crossing 1.0, 2.0, and $4.0 \mathrm{~nm}$ of $\mathrm{HfO}_{2}$. The narrow peak near $\Delta E=0$ for the shortest path corresponds to individual electronic excitations (including ionization) of an atom in the target. One notices that with increasing path length (i.e., number of atomic collisions), the Gaussian approximation (dotted lines) approaches the data from first principles calculations.

It has been shown ${ }^{22}$ that within the experimental resolution of current energy analyzers, an exponential decay function given by $f(\Delta E)=H(\Delta E) \alpha e^{-\alpha \Delta E}$, where $H$ denotes the Heaviside step function, reproduces ab initio energy loss calculations for near-zero impact parameter. We now show that this expression holds after averaging over all impact parameters, with significant improvement in accuracy over the Gaussian approximation and essentially the same convenience for practical applications.

The functional form of $f(\Delta E)$ given above is particularly convenient because it yields an analytical expression for its self-convolutions as well,

$$
f^{* n}(\Delta E)=\frac{\alpha^{n} E^{n-1}}{(n-1) !} e^{-\alpha \Delta E} H(\Delta E)
$$

Furthermore, once these self-convolutions are computed and Poisson statistics is taken into account [Eq. (1)], the energy loss distribution itself has a closed-form solution,

$$
F(\Delta E, x)=\frac{\lambda e^{(-m x-\alpha \Delta E)} I_{1}(2 \sqrt{\lambda \Delta E})}{\sqrt{\lambda \Delta E}} H(\Delta E)+e^{-m x \Delta E} \delta(\Delta E),
$$

where $I_{1}$ is the modified Bessel function of the first kind and $\lambda=m x \alpha ; m$ and $\alpha$ are related to the stopping power and straggling constant according to $\alpha=\left[2 /\left(d W^{2} / d x\right)\right](d E / x)$ and $m=\alpha(d E / d x)$. Plots of Eq. (3) are presented as solid lines in Fig. 1 and clearly show improvement over the Gaussian approximation, as they reproduce very closely $a b$ initio results. We will later show that once instrumental effects are taken into account, the deviation between this analytical approach and $a b$ initio calculations becomes negligible.

The energy spectrum of backscattered ions is determined by $F(\Delta E, x)$ and two additional effects that take place during Downloaded 26 Apr 2008 to 134.30.101.71. Redistribution subject
TABLE I. Scattering function $\chi_{i}$ considered for the calculations shown in Figs. 2 and 3.

\begin{tabular}{cc}
\hline \hline Model & Scattering function \\
\hline This work & $H\left(k_{i} E_{b}-E_{c}\right) \frac{1}{\Omega_{i}} e^{-\left(1 / \Omega_{i}\right)\left(k_{i} E_{b}-E_{c}\right)}$ \\
Ab initio & Numeric ${ }^{\mathrm{a}}$ \\
Gaussian & $\delta\left(k_{i} E_{b}-E_{c}\right)$ \\
\hline \hline
\end{tabular}

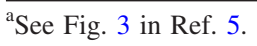

the scattering event: (i) the well-known energy transfer to the target nucleus, quantified by a kinematic factor $k$ that can be analytically expressed ${ }^{18}$ and (ii) the above mentioned, formerly overlooked inelastic energy loss due to electronic excitation of the backscattering target atom. The latter becomes more relevant with increasing atomic number of the target element, and its evaluation becomes mandatory for quantitative depth profiling of elements such as $\mathrm{La}, \mathrm{Hf}, \mathrm{Sb}$, and $\mathrm{Zr}$, commonly found in advanced and prototype electronic devices. These effects are incorporated in the calculations of Ref. 5 through convolution with a distribution function $\chi_{i}\left(k_{i} E_{b}-E_{c}\right)$, where $E_{b}$ and $E_{c}$ represent the ion energy before and after the atomic collision, respectively. In Ref. 5, $\chi_{i}\left(k_{i} E_{b}-E_{c}\right)$ is determined ab initio, whereas here, it is approximated by the exponential decay function proposed above for $f(\Delta E)$, where $\Omega_{i}$ is the variance of $\chi_{i}{ }^{22}$ Table I shows how the different models under consideration treat the backscattering event.

Figure 2 shows simulated $\mathrm{H}^{+}$scattering yields from $\mathrm{Hf}$ delta layers at different depths in $\mathrm{HfO}_{2}$ matrix according to the different energy loss models. Notice that once the energy loss due to the backscattering event is properly accounted for, there is excellent agreement between the proposed model and $a b$ initio calculations. Results shown below using the present approach make use of $\Omega_{\mathrm{Hf}}=187 \mathrm{eV}$ from ab initio calculations. A computationally straightforward estimation ${ }^{23}$ yields $\Omega_{\mathrm{Hf}}=217 \mathrm{eV}$ for a $\mathrm{H}^{+}$projectile at $100 \mathrm{keV}$ scattered by Hf.

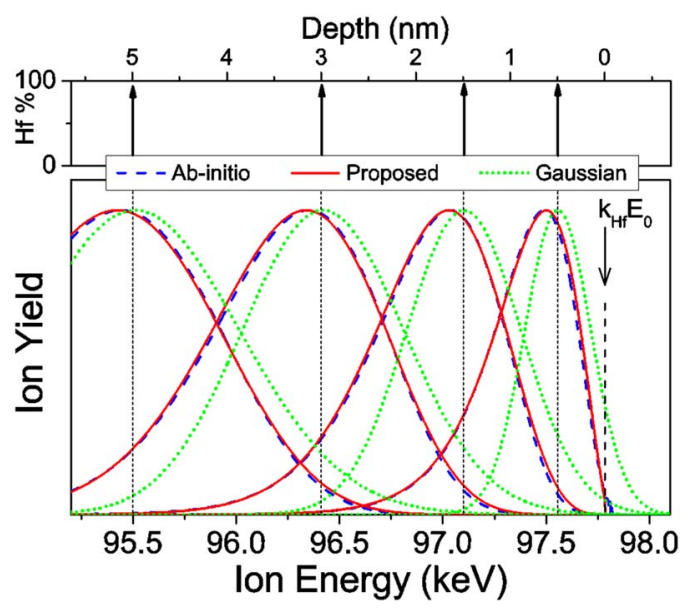

FIG. 2. (Color online) Ion scattering yield from $\mathrm{Hf}$ delta layers in a $\mathrm{HfO}_{2}$ matrix at $0.5,1.5,3.0$, and $5.0 \mathrm{~nm}$ from the surface as determined by $a b$ initio calculations (dashed lines), Eq. (3) (solid lines), and the standard Gaussian approximation (dotted lines). The curves are normalized to the same height for clarity. 


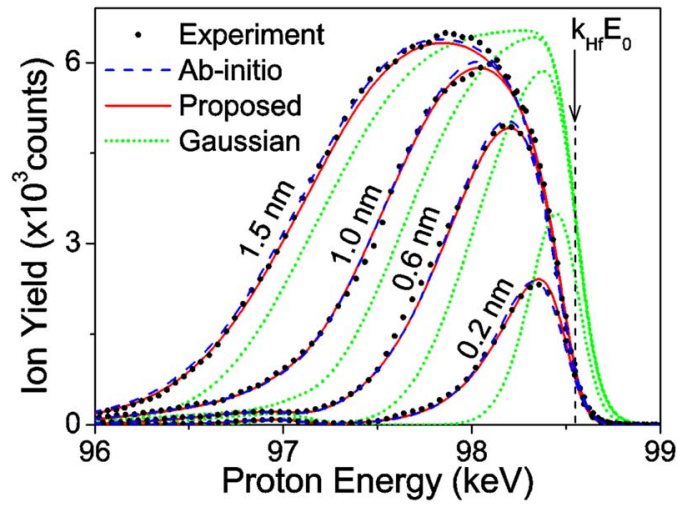

FIG. 3. (Color online) Experimental (symbols) and simulated (lines) MEIS spectra corresponding to ultrathin $\mathrm{HfO}_{2}$ films on $\mathrm{Si}$ acquired with $100 \mathrm{keV}$ $\mathrm{H}^{+}$ions at normal incidence and detected at a scattering angle of $110^{\circ}$.

The approach here proposed was also used to simulate experimental MEIS data acquired with $100 \mathrm{keV} \mathrm{H}^{+}$from ultrathin $\mathrm{HfO}_{2}$ films on $\mathrm{Si}$ that were known to be laterally homogeneous and form an abrupt interface with the substrate. ${ }^{24}$ In addition to the ion energy loss effects discussed above, these simulations take into account the following [see Eq. (10) in Ref. 5]: (i) the Rutherford scattering cross section, (ii) the depth distribution of the target element, and (iii) an instrumental (spectrometer) response function. Figure 3 shows the experimental data and simulations assuming $\mathrm{HfO}_{2}$ layers on $\mathrm{Si}$ whose thicknesses are the only free parameters. The instrumental function is a Gaussian of $180 \mathrm{eV}$ full width at half maximum. The quoted thicknesses were obtained from simulations based on ab initio energy loss calculations. These thicknesses were then used as inputs in the simulations based on the present approach and the Gaussian approximation. Excellent agreement is found among experimental data, ab initio calculations, and the results of this work. We have also applied the present approach to simulate MEIS data acquired with $200 \mathrm{keV} \mathrm{H}^{+}$from $\mathrm{TiO}_{2}$ films on Si (not shown). Simulations based on the Gaussian approximation can only be made to agree with the experimental data if intermixing between the oxide layers and $\mathrm{Si}$ is assumed. Hence, an oversimplification in the analysis may yield a completely wrong physical picture. The corrections introduced in the proposed model are also relevant for the recently developed technique of high resolution Rutherford backscattering spectrometry (as noted in Ref. 5) and, given their fundamental nature, any high resolution depth profiling method based on ion scattering.

In summary, we introduced an analytical expression for ion energy loss distributions which can substantially simplify the simulation of MEIS spectra in near-surface regions, where subnanometric depth resolution is required. The present approach avoids extensive, time consuming numerical ab initio calculations which are necessary to accurately take into account the ion energy lost for electronic excitation of the backscattering target atom. The inputs are the stopping power and straggling constant of the $\mathrm{H}^{+}$ion/target material pair at the energy of interest and a parameter related to electronic excitation of the target atom during the backscattering event. All these three parameters are readily available (with variable degree of accuracy) for elemental and compound targets. $^{20,21,23}$ The spectral simulations derived from the present approach showed excellent agreement with both $a b$ initio calculations and experimental MEIS results.

The authors would like to acknowledge Matt Copel for fruitful discussions and providing the experimental data, Nuno Barradas for proofreading the manuscript, and financial support from the Brazilian agencies MCT/CNPq, CAPES, and FAPERGS.

${ }^{1}$ R. G. Smeenk, R. M. Tromp, H. H. Kersten, A. H. J. Boerboem, and F. W. Saris, Nucl. Instrum. Methods Phys. Res. 195, 581 (1982).

${ }^{2}$ J. F. Ziegler, J. P. Biersack, and U. Littmark, The Stopping and Range of Ions in Solids (Pergamon, New York, 1985).

${ }^{3}$ J. F. van der Veen, Surf. Sci. Rep. 5, 199 (1985).

${ }^{4}$ M. Copel, IBM J. Res. Dev. 44, 571 (2000).

${ }^{5}$ R. P. Pezzi, P. L. Grande, M. Copel, G. Schiwietz, C. Krug, and I. J. R. Baumvol, Surf. Sci. 601, 5559 (2007).

${ }^{6}$ G. Schiwietz, Phys. Rev. A 42, 296 (1990).

${ }^{7}$ P. L. Grande and G. Schiwietz, Theory of the Interaction of Swift Ions with Matter, Advances in Quantum Chemistry Vol. 45 (Academic, Amsterdam, 2004) Pt. 1, Chap. 2, pp. 7-46.

${ }^{8}$ I. Vickridge and G. Amsel, Nucl. Instrum. Methods Phys. Res. B 45, 6 (1990).

${ }^{9}$ G. Amsel, Nucl. Instrum. Methods Phys. Res. 194, 1 (1982).

${ }^{10}$ B. Maurel, G. Amsel, and J. P. Nadai, Nucl. Instrum. Methods Phys. Res. 197, 1 (1982).

${ }^{11}$ P. Sigmund, Particle Penetration and Radiation Effects (Springer, Berlin, 2006).

${ }^{12}$ C. J. Yim, D. Ko, M. H. Jang, K. B. Chung, M. Cho, and H. T. Jeon, Appl. Phys. Lett. 92, 012922 (2008).

${ }^{13}$ I. Kim, S. Kuk, J. Kim, H. Jeon, M. Cho, and K. B. Chung, Appl. Phys. Lett. 90, 222101 (2007).

${ }^{14}$ C. Maunoury, K. Dabertrand, E. Martinez, M. Saadoune, D. Lafond, F. Pierre, O. Renault, S. Lhostis, P. Bailey, T. C. Q. Noakes, and D. Jalabert, J. Appl. Phys. 101, 034112 (2007).

${ }^{15}$ L. V. Goncharova, M. Dalponte, D. G. Starodub, T. Gustafsson, E. Garfunkel, P. S. Lysaght, B. Foran, J. Barnett, and G. Bersuker, Appl. Phys. Lett. 89, 044108 (2006).

${ }^{16}$ R. Barnes, D. Starodub, T. Gustaffson, and Garfunkel, J. Appl. Phys. 100, 044103 (2006).

${ }^{17}$ E. P. Gusev, H. C. Lu, T. Gustafsson, and E. Garfunkel, Phys. Rev. B 52, 1759 (1995).

${ }^{18}$ W.-K. Chu, J. W. Mayer, and M.-A. Nicolet, Backscattering Spectrometry (Academic, New York, 1978).

${ }^{19}$ H. W. Schulte, B. W. Busch, E. Garfunkel, T. Gustafsson, G. Schiwietz, and P. L. Grande, Nucl. Instrum. Methods Phys. Res. B 183, 16 (2001).

${ }^{20} \mathrm{~J}$. F. Ziegler, SRIM, The stopping and range of ions in matter, 2006 (http:// www.srim.org).

${ }^{21}$ W. K. Chu, Phys. Rev. A 13, 2057 (1976).

${ }^{22}$ P. L. Grande, A. Hentz, R. P. Pezzi, I. J. R. Baumvol, and G. Schiwietz, Nucl. Instrum. Methods Phys. Res. B 256, 92 (2007).

${ }^{23} \mathrm{P}$. L. Grande and G. Schiwietz, CASP, Convolution approximation for swift particles, 2006 (http://www.hmi.de/people/schiwietz/casp.html).

${ }^{24}$ E. P. Gusev, C. Cabral, Jr., M. Copel, C. D’Emic, and M. Gribelyuk, Microelectron. Eng. 69, 145 (2003). 